\title{
A novel method for computation of temperature profile of turbine disk
}

\author{
Weilin DONG*, Suofang WANG* and Jiajia NIU* \\ *College of Energy and Power Engineering, Nanjing University of Aeronautics and Astronautics, Nanjing 210016, China \\ E-mail: sfwang@nuaa.edu.cn
}

Received: 25 September 2017; Revised: 30 October 2017; Accepted: 10 December 2017

\begin{abstract}
A novel temperature computation method of turbine disk is proposed according to theoretical analysis, where the coupling analysis of the outer surface in disk and fluid relative to this outer surface was carried out, instead of the segregation condition (constant wall temperature or constant wall heat flux condition). The selected correlations, numerical and experimental data of the heat transfer coefficient are presented and compared, so are the theoretical and simulated temperature profiles. The results show that: The maximum temperature deviation coefficient $\left(\varphi_{T}\right)$ between the theoretical results and simulation data is less than $8.7 \%$ while $R e_{\Phi}=$ $4.65 \times 10^{5}, C_{w}=7520, R e_{m}=5 \times 10^{5}$. The convective heat transfer coefficient distributions at the disk outer surface exhibited the influence of region A and region $\mathrm{B}$. The convective heat transfer is dominated by the mass flow rate of cooling air in region $\mathrm{A}$, while dominated by the mainstream mass flow rate in region $\mathrm{B}$. The temperature of the turbine disk decreases with the mainstream mass flow rate increasing, especially at the high radius of turbine disk, while the overall temperature of turbine disk decreases with the increase of cooling air mass flow rate. Both the $h_{r}$ and $h_{z}$ increase with the $R e_{\Phi}$ increasing, leading to a minor increase in temperature of turbine disk eventually.
\end{abstract}

Keywords : Turbine disk, Cooling air, Convective heat transfer coefficient, Temperature profile, Coupling analysis

\section{Nomenclature}

$a \quad$ thickness of turbine disk (m)

$b \quad$ outer radius of turbine disk $=r_{e}(\mathrm{~m})$

$C_{w} \quad$ non-dimensional secondary air flow rate

$c_{p, m} \quad$ specific heat of mainstream $\left(\mathrm{J} \cdot \mathrm{kg}^{-1} \cdot \mathrm{K}^{-1}\right)$

$c_{p, c} \quad$ specific heat of cooling air $\left(\mathrm{J} \cdot \mathrm{kg}^{-1} \cdot \mathrm{K}^{-1}\right)$

$h \quad$ heat transfer coefficient $\left(\mathrm{W} \cdot \mathrm{m}^{-2} \cdot \mathrm{K}^{-1}\right)$

$\dot{m} \quad$ mass flow rate of cooling air $\left(\mathrm{kg} \cdot \mathrm{s}^{-1}\right)$

$\bar{M} \quad$ blowing ratio $=\left(\rho_{c} V_{c}\right) /\left(\rho_{m} V_{m}\right)$

$N u_{r} \quad$ local Nusselt number in side surface

$N u_{z} \quad$ local Nusselt number in outer surface

$Q_{r} \quad$ side surface heat transfer rate (W)

$Q_{z} \quad$ outer surface heat transfer rate (W)

$r_{i} \quad$ inner radius of turbine disk (m)

$r \quad$ radial coordinate $(\mathrm{m})$

$R e_{m} \quad$ mainstream flow Reynolds number

$R e_{\Phi} \quad$ rotational Reynolds number

$R e_{r} \quad$ local rotational Reynolds number

$R e_{c} \quad$ cooling air flow Reynolds number $=\rho V_{c} S_{c} / \mu$
$T_{\text {ref }} \quad$ reference temperature of cooling air (K)

$T_{w, r} \quad$ turbine disk side surface temperature (K)

$T_{w, z} \quad$ turbine disk outer surface temperature (K)

$T_{a w} \quad$ reference temperature of mainstream (K)

$V_{m} \quad$ mainstream flow bulk axial velocity $\left(\mathrm{m} \cdot \mathrm{s}^{-1}\right)$

$V_{c} \quad$ cooling air flow bulk radial velocity $\left(\mathrm{m} \cdot \mathrm{s}^{-1}\right)$

$z \quad$ axial coordinate $(\mathrm{m})$

$Z \quad$ non-dimensional axial coordinate $=z / a$

Greek letter

$\varphi_{T} \quad$ temperature deviation coefficient

$\omega \quad$ rotational angular velocity $\left(\mathrm{rad} \cdot \mathrm{s}^{-1}\right)$

$\rho \quad$ fluid density $\left(\mathrm{kg} \cdot \mathrm{m}^{-3}\right)$

$\lambda \quad$ fluid thermal conductivity $\left(\mathrm{W} \cdot \mathrm{m}^{-1} \cdot \mathrm{K}^{-1}\right)$ Subscripts

c cooling air

$i \quad$ inner

$m$ mainstream

ref reference 
$S \quad$ axial gap between rotor and stator (m)

$S_{c} \quad$ seal clearance (m)

$T_{t} \quad$ theory temperature (K)

$T_{s} \quad$ simulation temperature $(\mathrm{K})$

$T_{m} \quad$ mainstream inlet temperature $(\mathrm{K})$

$T_{c} \quad$ cooling air inlet temperature $(\mathrm{K})$

$\begin{array}{ll}r & \text { local radius } \\ s & \text { simulation } \\ t & \text { theory } \\ T & \text { temperature } \\ w & \text { wall } \\ z & \text { axial distance }\end{array}$

\section{Introduction}

Turbine disk, which is loaded down with the centrifugal stress and the thermal force caused by the high rotational speed and the non-uniform temperature field respectively, is one of the most important components in the aero engine. Thus, it was defined as a life limited part by Federal Aviation Administration (FAA). Fig. 1 illustrates a typical rotor-stator disk cavity with rim seal, heat transfers radially inward from the outer surface of turbine disk; cooling air cools the turbine disk side surface, then it mixes with the hot gas at the exit of the rim seal, Besides, it isolates the heat from the hot gas at the outer surface just like "Film cooling effect".

One of the main purposes of the cooling air is to keep the temperature of the turbine disk in a reasonable range. However, too much cooling air exacts a penalty on turbine cycle performance. Thus, efficient use of cooling air requires a good understanding of the heat transfer characteristic and the temperature calculation model.

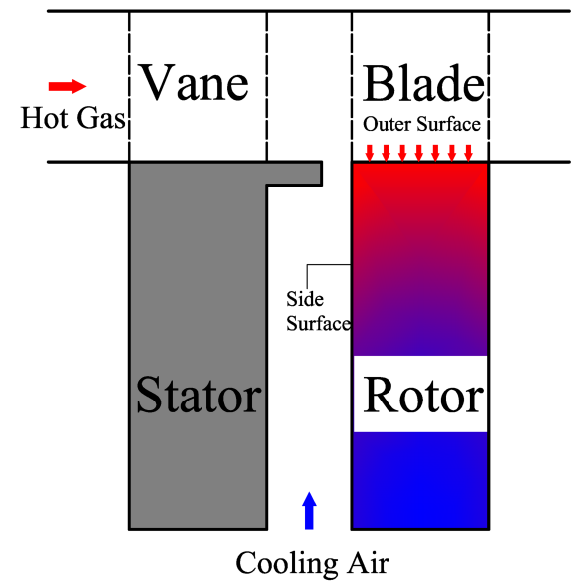

Fig.1 A typical rotor-stator cavity

\subsection{Heat transfer}

In the earlier years, researches on the flow and heat transfer characteristics of the turbine disk mainly focused on the heat transfer coefficient distribution of the turbine disk side surface. To make the results more universal, the general practice is to simplify the turbine disk to the uniform thickness disk (rotor disk). The first insight to the flow characteristics of free rotor disk was conducted by Karman (1921). The qualitative results of the disk resistance moment $(M)$ and boundary layer thickness $(\delta)$ was obtained. Adding a stator disk on the side of rotor disk can change the flow characteristics near to the rotor disk. Batchelor (1951) believe that the surfaces of the rotating disk and static disk have their own independent boundary layers. There is a fluid core, whose rotational speed $(\omega c)$ is $10 \sim \omega$ between the two disks. While another flow structure was proposed by Stewartson (1953). As argued in the paper, a boundary does exist on the surface of the rotating disk. However, the tangential velocity of the fluid decreases from $\omega r$ (near the rotor disk) to 0(near the stator disk). Daily and Nece (1960) studied the flow characteristics in the rotor-stator cavity, and validated that the two kinds of flow structures do exist. Furthermore, they divided the flow structures in the rotor-stator cavity into four regions with different $G$ and $R e_{\Phi}$. Owen and Bayley (1974) and Haynes and Owen (1975) divided the rotor-stator cavity flow region into source region and core region according to the level of the radius (the former's radial velocity and the latter's tangential velocity are zero).

In the field of numerical simulation, Chew (1984) was the first to use a k- $\varepsilon$ turbulence model to investigate the flow 
characteristics in a rotor-stator cavity. Kilic (2001) numerically studied the effect of gap ratio, Reynolds number and the mass flow rate on the flow and heat transfer structure inside isothermal and heated rotating cavities with peripheral flow of cooling air. Pocent and Schiestel (2007) utilized the Reynolds stress model (RSM) to simulate the flow characteristics inside three different rotor-stator cavities, and the results were in satisfactory agreement with the experimental data. A combined numerical and experimental study was performed by Yuan et al. (2003) to determine the turbulent heat transfer in a rotor-stator cavity by the means of RNG k- $\varepsilon$ model and the steady-state liquid crystal technique in the range of the $R e_{\Phi}$ from $1.42 \times 10^{5}$ to $3.33 \times 10^{5}$. In his paper, an optimum rotor-stator distance for a given $R e_{\Phi}$, where the average heat transfer on the stator reaches maximum. The heat characteristics of rotor-stator cavities with ingestion from hot gas was studied by Wang and Wilson (2012). They numerically analyzed the flow and heat transfer characteristics in a rotor-stator cavity, and discovered that the flow structures had been changed by the ingestion from the hot gas which carry with a large angular momentum.

In experimental aspect, the technique of infrared radiation (IR) (Astarita and Cardone, 2008; Fenot et al., 2005; Pelle and Harmand, 2007,2009) was utilized extensively to measure the convective heat distributions. Besides, Bunker (1992) used transient liquid crystal (TLC) to measure convictive heat transfer coefficient radial distribution on a rotor disk side surface in a rotor-stator cavity where cooling air was supplied at the cavity hub, They found out that the heat transfer was affected by the impact of the fluid and the rotational speed at low radius and high radius respectively. The technique of TLC was also used by Luo et al. (2014) to explore the flow structure and heat transfer characteristics in a rotor-stator cavity with inlet at low radius. Chen et al. (1998) experimentally investigated the heat transfer characteristics of a rotor disk with an impinging circular jet by the means of naphthalene sublimation, and found that the heat transfer can be divided into three regimes, namely the impingement dominated regime; the mixed regime and the rotation dominated regime.

\subsection{Temperature profile}

On the other hand, some scholars have made considerable efforts to calculate the temperature distribution of turbine disk. Ding et al. (2013) assumed the outer face of turbine disk as second thermal boundary condition, basing on a series of empirical heat transfer formulas on the turbine disk side surface. They obtained the One-dimensional temperature distribution of turbine disk, which is the function of the first order Bessel function and zero order Bessel function. Also, Lin et al. (2015) employed the theoretical analysis methods to obtain the temperature distribution of turbine disk. In comparison with the assumption of Ding, they used first thermal boundary condition on the outer face of turbine disk to obtain the temperature distribution of turbine disk, simplified the rectangular coordinates to the cylindrical coordinates, and found out that only at relatively small $B i$ can it make difference between the results of the two coordinate systems.

Previous studies have obtained a large number of results concerning the heat transfer characteristics on the turbine disk surface. However, as the fact that cooling air can isolate the heat from the hot gas on the turbine disk outer surface haven't been fully considered, it is still relatively lacking of the exactly conversion approach of these heat transfer characteristics to the turbine disk temperature distribution. This paper proposes a novel method of the temperature computation model of turbine disk which is different from the conventional model as shown in Fig 2. This temperature computation model utilizes the coupling condition instead of the segregation condition, which means that the thermal isolation effects of the cooling air were firstly taken into account in this study. It should be noted that the heat transfer coefficient value is directly related to the reference temperature, To the author's knowledge, there are 3 temperatures used as the reference temperature in the most of studies - the cooling air inlet temperature, the adiabatic temperature on the rotor disk surface and the core fluid temperature. In this study, the cooling air inlet temperature was employed as the reference temperature considering the convenience of theoretical calculation. 


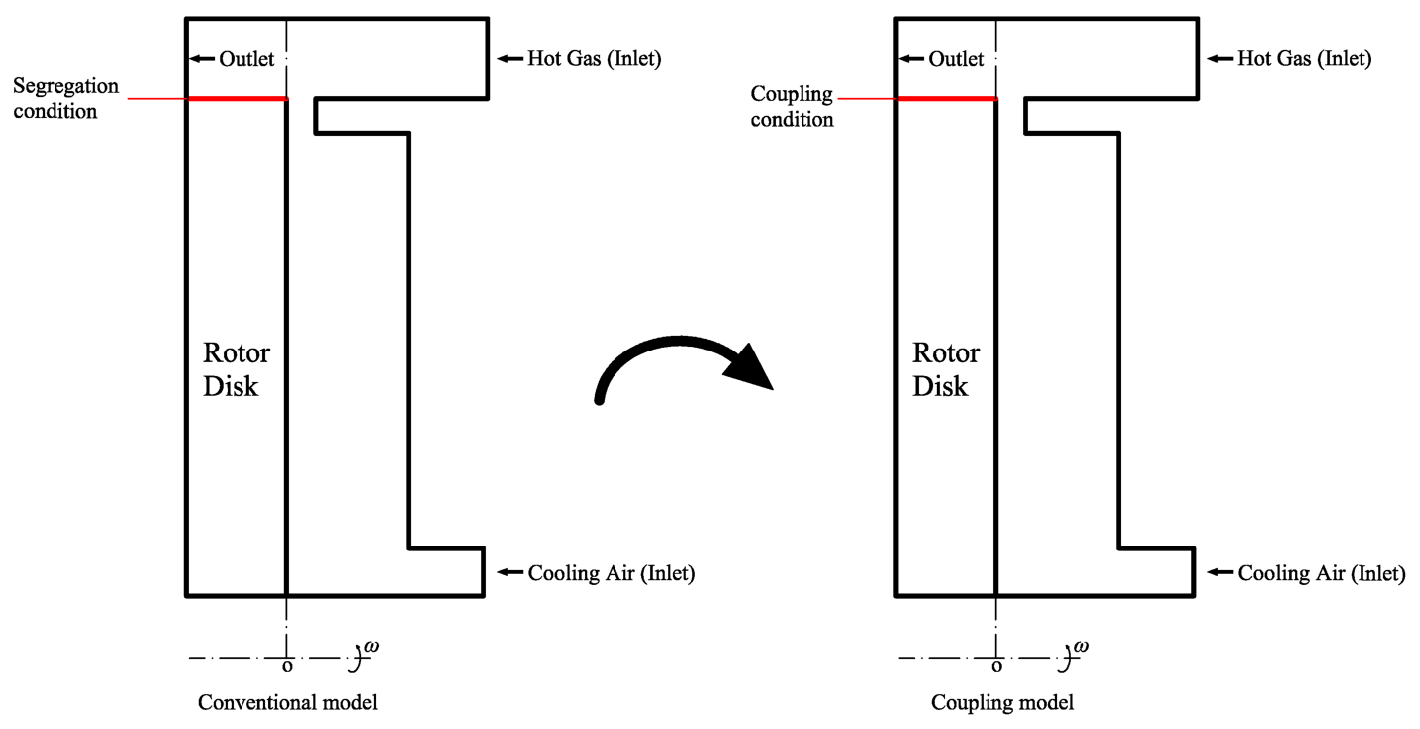

Fig. 2 Two types of temperature computation models in turbine disk

To utilize the numerical results and some experiments data (Roy et al., 2003) to validate the feasibility and validity of a temperature coupling model of turbine disk, the heat transfer and temperature distribution characteristics of turbine disk have been analyzed and discussed. The temperature coupling model in this study can lay a foundation for the design of the turbine, especially for the thermal stress analysis of turbine disk.

\section{Heat transfer analysis}

In this section, the heat transfer characteristics of the turbine disk, including the side surface and out surface, is described. The results can provide the basis for calculation of surface temperature for turbine disk.

\subsection{Heat transfer on side surface}

A convective heat transfer correlation (Roy et al., 2003) had been proposed. Note that the correlation has their corresponding working condition ranges. This paper utilizes a formula which is from Roy, and it can be expressed as:

$$
N u_{r}(r)=0.0195 R e_{r}^{0.8}
$$

Thus, the local heat transfer coefficient $h_{r}(\mathrm{r})$ can be written as:

$$
h_{r}(r)=\frac{N u_{r} \lambda_{\text {air }}}{r}=0.195 \lambda_{\text {air }}\left(\frac{\rho \omega}{\mu}\right)^{0.8} r^{0.6}
$$

The working condition ranges of the Eq. (2) are: $4.65 \times 10^{5}<R e_{\phi}<8.6 \times 10^{5}, 1504<C_{w}<7520$. In fact, changing $C_{w}$ can affect the calculation accuracy of the equation (2). The average deviation of the equation (2) is $4.1 \%$ for $C_{w}=$ 1504 and $12.2 \%$ for $C_{w}=7520$.

As a result, the heat transfer rate on the turbine disk side surface $Q_{r}$ can be express as

$$
Q_{r}=\int_{0}^{2 \pi} \int_{0}^{r_{e}} h(r)\left(T_{w}(r)-T_{r e f}(r)\right) r d r d \theta
$$

where $T_{w}(\mathrm{r})$ is local wall temperature and $T_{\text {ref }}$ is fluid reference temperature (cool air inlet temperature).

\subsection{Heat transfer on outer surface}

How to describe the flow and heat transfer on the turbine disk outer surface is always a difficult problem. Essentially, the flow types on the turbine disk outer surface have a level of similarity to the film cooling, Metzger et al. 
(1968) proposed their heat transfer empirical formula which have a strict working condition range, thus, that formulas can't be directly employed in this paper. Nevertheless, that correlations' form are similar, it can be correlated by a relation of the form:

$$
N u_{z}(z)=a\left(\operatorname{Re}_{c} \frac{x}{S_{c}}\right)^{b}
$$

Note that Eq. (4) is from cooling film, however, there is an obvious difference between the cooling film and this paper's model, that is, this paper's model have a high rotational speed, consequently, taking the rotation effect into account, we revise the Eq. (4) to

$$
N u_{z}(z)=a\left(\operatorname{Re}_{c} \frac{x}{S_{c}}\right)^{b} R e_{\Phi}^{c}
$$

According to the Eq. (5), the local convictive heat transfer coefficient can be written as

$$
h_{z}(z)=c_{1}\left(\operatorname{Re}_{c} \frac{x}{S_{c}}\right)^{c_{2}} \lambda_{c}\left(\operatorname{Re}_{\Phi}\right)^{c_{3}} / z
$$

where $c_{1}, c_{2}$ and $c_{3}$ can be obtained in the Sec.6 by using fitting method. So the heat on the turbine disk side surface $Q_{z}$ can be expressed as:

$$
Q_{z}=2 \pi r_{e} \int_{0}^{a} h_{z}(z)\left(T_{a w}(z)-T_{w}(z)\right) d z
$$

It should be noted that $T_{a w}(\mathrm{z})$ is the reference temperature (adiabatic wall temperature in the cooling film ), and it could be expressed as follow:

$$
T_{a w}(z)=T_{g}+\frac{T_{c}-T_{g}}{1+\left(\frac{Z}{\bar{M}}\right) \frac{c_{\rho g}}{c_{\rho c}}}
$$

\section{Derivation of the disk temperature}

The heat transfer characteristics on the side and outer surface of turbine disk have been discussed in last section. In this section, we turn the third thermal boundary condition into first thermal boundary condition using the energy conservation equation and the Iterative method, then employ the acquired first thermal boundary condition to calculate the temperature distribution of turbine disk.

\subsection{Temperature distribution calculation}

As the axial length of the turbine disk is much smaller than the radial length, the disk temperature is assumed to be axially constant. Then the thermal conduction differential equation can be expressed as

$$
\frac{d^{2} T(r)}{d r^{2}}+\frac{1}{r} \frac{d T(r)}{d r}=\frac{h_{a v}(r)}{\lambda \delta}\left(T(r)-T_{r e f}\right)
$$

where

$$
r=r_{i}, \frac{d T(r)}{d r}=0 ; r=r_{o}, \mathrm{~T}(r)=T_{o}
$$

The general solution of equation (10) is:

$$
T(r)=C_{1} I_{0}(m r)+C_{2} K_{0}(m r)+T_{r e f}
$$

Where $I_{0}(m r), K_{0}(m r)$ are zero order Bessel function, $C_{1}, C_{2}$ are undetermined coefficients. Substituting Eq. (10) into equation (11) then gives 


$$
\begin{aligned}
& C_{1}=\left[\left(T_{e}-T_{r e f}\right) K_{1}\left(m r_{i}\right)\right] /\left[I_{0}\left(m r_{e}\right) K_{1}\left(m r_{i}\right)-I_{1}\left(m r_{i}\right) K_{0}\left(m r_{e}\right)\right] \\
& C_{2}=\left[\left(T_{e}-T_{r e f}\right) I_{1}\left(m r_{i}\right)\right] /\left[K_{0}\left(m r_{e}\right) I_{1}\left(m r_{i}\right)-K_{1}\left(m r_{i}\right) I_{0}\left(m r_{e}\right)\right]
\end{aligned}
$$

Finally, $C_{1}, C_{2}$ are obtained which means that the expression of turbine disk temperature distribution is acquired. It should be noted that $T_{o}$ is undetermined value which will be determined in the next section.

\subsection{Thermal boundary analysis}

In models, the other disk side surface is assumed to be an adiabatic wall, thus, the energy conservation equation of turbine disk including $T_{o}$ can be express as:

$$
Q_{r}=\int_{0}^{2 \pi} \int_{0}^{r_{e}} h(r)\left(T(r)-T_{r e f}\right) r d r d \theta=Q_{z}=2 \pi r_{e} \int_{0}^{a} h_{z}(z)\left(T_{a w}(z)-T_{o}\right) d z
$$

Where the expressions of $h_{r}(r), h_{z}(z)$ can be seen in Eq. (2), Eq. (5)respectively. However, $T_{o}$ could not be expressed with the elementary function because the definite integral in Eq. (14) could not be solved. Hence, Newton Rafael iterative method was used to solve the Eq. (14) where steps are:

Define $\Delta Q$ as :

$$
\Delta Q=Q_{z}-Q_{r}
$$

First, set $T_{o}=T_{c}$, and then substitute $T_{o}$ into Eq. (14), finally, the corresponding $\Delta Q$ was acquired.

Make $F^{\prime}=d(\Delta Q) / d T$, so $T_{o}$ can be modified as:

$$
T_{o}=T_{o 1}=T_{c}-\Delta Q / F^{\prime}
$$

After modifying $T_{o}$ to $T_{o l}$, take the second iteration, then loop the steps above until $\Delta Q$ is less than the set of residual $\varepsilon$, which was set as $1 \times 10^{-1}$. At this point, the temperature distribution of turbine disk can be obtained.

\section{Computational model and approach}

To analyze and validate the temperature distribution above, this section introduces the pertinent computational model and boundary condition.

\subsection{Geometry model and mesh partition}

The commercial CFD code CFX-15 is employed in this paper, the mainly geometry parameters of disk are same as the literature's published by Roy et al. (2001). It should be noted that this paper focus on the heat transfer and temperature distribution characteristics while the sealing effects have not been much discussed, consequently, Roy's model has been simplified as shown in Fig. 3, where the outer sealing ring was neglected as same as the vanes and blades. 


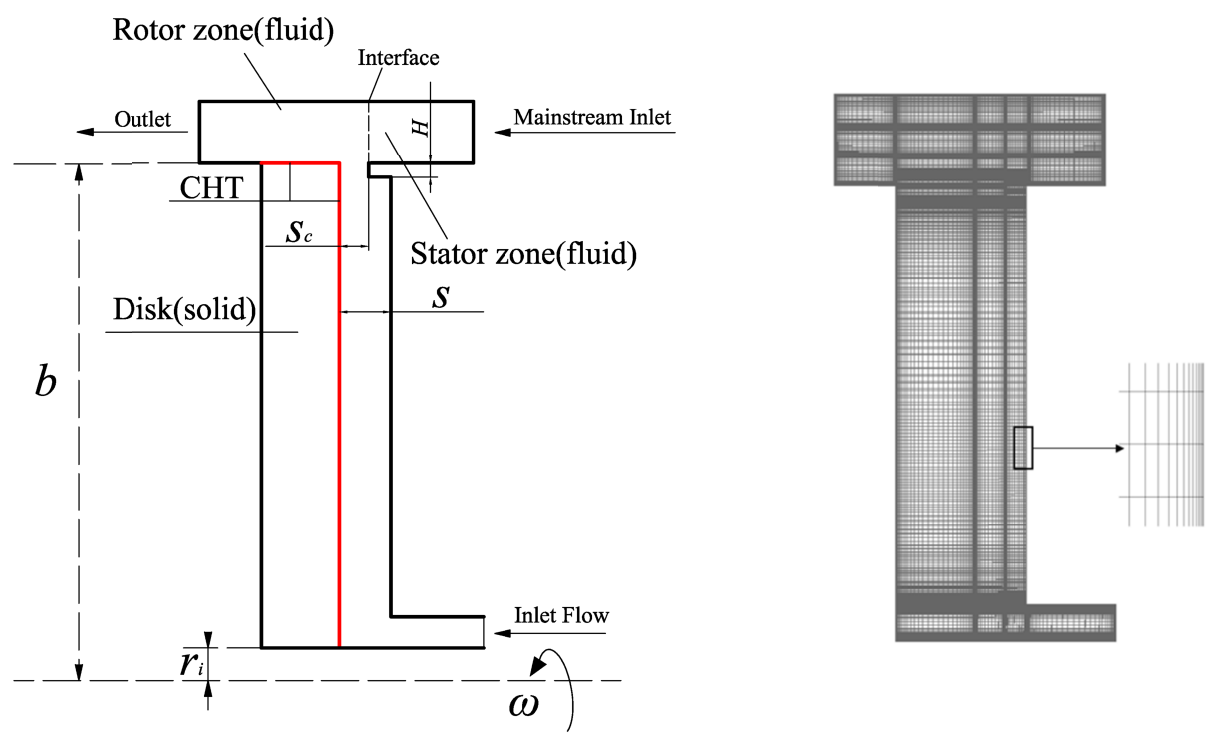

Fig. 3 Illustration of computational geometry and mesh

To reduce the set-up time, a hexahedral mesh produced by ICEM-CFD has been adopted in the numerical simulations as depicted. Two kinds of near-wall grid spacing of $0.005 \mathrm{~mm}$ and $0.002 \mathrm{~mm}$ were employed, where the one of $0.002 \mathrm{~mm}$ could not be converged in the numerical simulations because of the excessive aspect ratio. Conversely, the one of $0.005 \mathrm{~mm}$ shows a good convergence, additionally, it satisfies the condition of the near-wall $y+\approx 1$. After the grid independence verification, the final grid number is 2100000 .

\subsection{Boundary conditions}

No-slip wall condition was applied in model. Additionally, the conjugate heat transfer (CHT), which means the heat flux conversation, is performed in interface between fluid and solid domain. As the residuals of energy equation and momentum equation are less than $1 \times 10^{-5}$ and $1 \times 10^{-6}$ respectively, the computed results are considered convergent.

In this paper, both the mainstream inlet and the cooling air inlet are specified as mass flow inlet while the mainstream outlet was set as pressure outlet. According to the previous studies, the mass flow rates of the mainstream inlet and cooling air inlet can be dimensionless as $R e_{m}$ and $C_{w}$ respectively, furthermore, the disk rotational speed $(\omega)$ is dimensionless as $R e_{\Phi}$. All of the dimensionless parameters above can be written as:

$$
\begin{aligned}
& C_{w}=\dot{m} / \mu b \\
& R e_{\Phi}=\rho \omega b^{2} / \mu \\
& R e_{m}=\rho V_{m} b / \mu
\end{aligned}
$$

Where $\dot{m}$ is cooling air mass flow rate, $V_{m}$ represents the velocity of the mainstream flow, $b$ denotes the outer radius of rotor disk and $\mu$ is the dynamic viscosity in the cooling air inlet. Table 1 provides the operating conditions in this study, where the parameter values in bold had been studied experimentally by Roy et al. (2001). And the others were only numerically done. Note that there can be the ingestion of hot gas under certain condition (small mass flow rate of cooling air and high rotation speed of rotor disk). However, it is beyond the scope showed in table 1 . Thus, the ingestion of hot gas has not been involved in this paper. In addition, there is no ingestion when the engine is working normally, therefore the research work in this paper focuses on the condition of no ingestion (the engine works normally). 
Table 1 Operating condition

\begin{tabular}{|c|c|c|}
\hline$C_{w}$ & $R e_{\Phi}$ & $R e_{m}$ \\
\hline $\begin{array}{l}1504 \text { (Exp.) } \\
3008 \text { (Exp.) } \\
7520 \text { (Exp.) }\end{array}$ & $4.65 \times 10^{5}($ Exp. $), 7.0 \times 10^{5}(\operatorname{Exp}),. 8.6 \times 10^{5}($ Exp. $)$ & $\mathbf{5 \times 1 0 ^ { 5 }}$ (Exp.), $7.5 \times 10^{5} \quad 1 \times 10^{6}$ \\
\hline
\end{tabular}

\section{Comparison and analysis of results}

Theoretical and simulation heat transfer characteristics are presented and compared in this section as well as temperature distributions. In the following discussion, the solid lines mean simulation, the dot dash lines correspond to theoretical result and the symbols represent experimental data.

Fig. 4(a-b) illustrates the convective heat transfer coefficient distributions at the turbine disk surface for different $R e_{\Phi}$, where the results of simulation, theoretical and experimental at the side surface of turbine disk are presented and compared in Fig. 4a. It can be seen that all of the lines reveal a similar conclusion: The convective heat transfer coefficient distribution at the rotor disk surface can be divided into two regions, the source region and the core region. In the source region, which is radially inboard, the convective heat transfer is dominated by the cooling air flow rate. In the core region, which is radially outboard, the heat transfer is dominated by the rotational speed. Compared with the experimental results, the simulation data shows encouraged good agreement at low radius, while the deviation increases with the radius increasing and it reaches the maximum value of $9.8 \%$ at $R e_{\Phi}=8.6 \times 10^{5}, r=0.17 \mathrm{~m}$. The using of cooling air inlet temperature as the reference temperature may be responsible for this discrepancy. Also, comparing the theoretical results with the experimental data, we can find that the two curves are in good agreement at high radius especially when $R e_{\Phi}$ is equal to $4.65 \times 10^{5}$, and the discrepancy increases with the $R e_{\Phi}$ increasing. Fig. $4 \mathrm{~b}$ depicts the convective heat transfer coefficient distributions at the turbine disk outer surface for different Reo, it can be concluded that $h_{z}$ is relatively insensitive to the change of $R e_{\Phi}$, nevertheless, rotational effect was considered to improve calculation accuracy, where the correlation has been expressed as Eq. (5), the maximum deviation from the experimental data to Eq. (5)'s value is not more than $9.7 \%$ at $c_{1}=88, c_{2}=-0.23, c_{3}=0.12$ for $4.65 \times 10^{5}<R e_{\Phi}<$ $8.6 \times 10^{5}$. Therefore, it is feasible to utilize the correlation of Eq. (5) to express the heat transfer at the turbine disk outer surface.

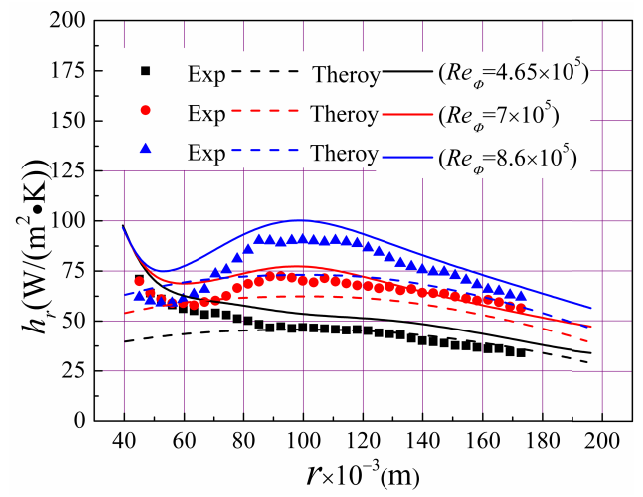

(a) Heat transfer on side surface

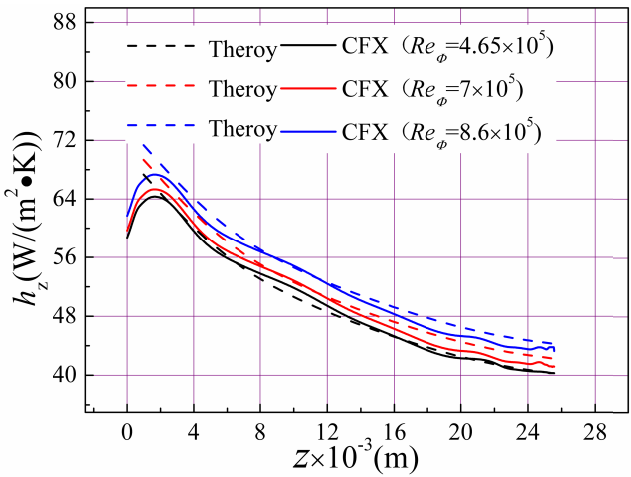

(b) Heat transfer on outer surface

Fig. 4 Effect of $R e_{\Phi}$ on the heat transfer distributions $\left(C_{w}=3008, R e_{m}=5 \times 10^{5}\right)$

Fig. 5(a-b) delineates the effect of $C_{w}$ on the heat transfer coefficient distributions at the turbine disk side surface and outer surface respectively. According to the Fig. 5a, the coefficient of heat transfer at low $\operatorname{radius}(r / b<0.59)$ changes with radius significantly while it does not differ by much at high $\operatorname{radius}(r / b \geq 0.59)$, which is opposite to Fig 4a's result. A same conclusion as fig.4a can be obtained, so no more detail will be given in this paragraph. Comparing the theoretical results with experimental data, it can be concluded that the theoretical results are in good agreement with experimental results at $C_{w}=1504$. Additionally, the discrepancy between two kinds of results increases while $C_{w}$ increases from 1504 to 7520 , which is caused by the independent variable of the correlation (as expressed in equation 
2) relating to the angular velocity $(\omega)$ but not the secondary air flow rates $\left(C_{w}\right)$. Observation of Fig. $5 \mathrm{~b}$ shows that the results of theoretical and experimental are in good agreement in the region near to the outlet of cooling air (region A, white region as shown in Fig. 5(b)), while there is an obvious discrepancy between two kinds of results in the region far from the outlet of cooling air (region B, gray region as shown in Fig. 5(b)). This is due to the full mixing of hot gas and cooling air at region B while it does not at the region B. In region B, the "film cooling effect" has largely disappeared, which means heat transfer is dominated by the hot gas flow rate. Note that it can be observed an interesting phenomenon, namely, the dividing line between region A and region B is not fixed, which will move toward direction of region A with the $C_{w}$ increasing.

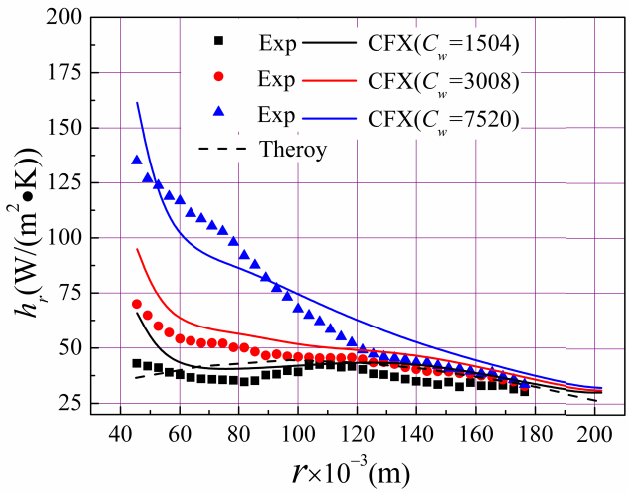

(a) Heat transfer on side surface

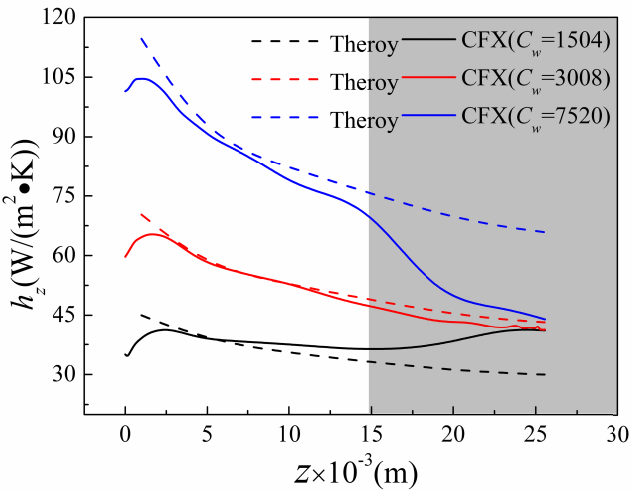

(b) Heat transfer on outer surface

Fig. 5 Effect of $C_{w}$ on the heat transfer distributions $\left(R e_{\Phi}=4.65 \times 10^{5}, R e_{m}=5 \times 10^{5}\right)$

Figures 6(a-b) illustrates the convective heat transfer coefficient distributions at the turbine disk surface for different $R e_{m}$. Fig. 6a plots the $h_{r}$ distributions at the disk side surface. There is a minor difference for different $R e_{m}$, as the maximum difference of heat transfer coefficient is less than $5.4 \%$ with the $R e_{m}$ increase from $5 \times 10^{5}$ to $1 \times 10^{6}$. Although this figure is lack of experimental verification, the conclusion that change of $R e_{m}$ has little effect on $h_{r}$ at disk side surface can be obtained. Inspection of Fig $6 \mathrm{~b}$ shows that it can also be divided into region $\mathrm{A}$ and region $\mathrm{B}$ which is same to Fig 5b, where the maximum deviation from the theoretical results to simulation results is $6.3 \%$ in region $\mathrm{A}$, and $25 \%$ in the region $\mathrm{B}$.

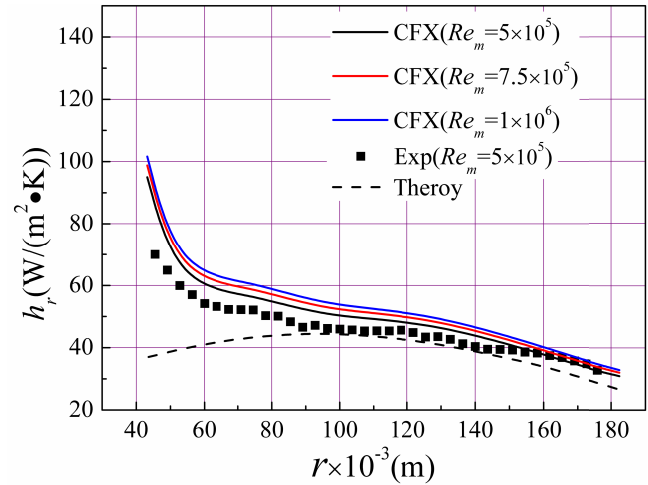

(a) Heat transfer on side surface

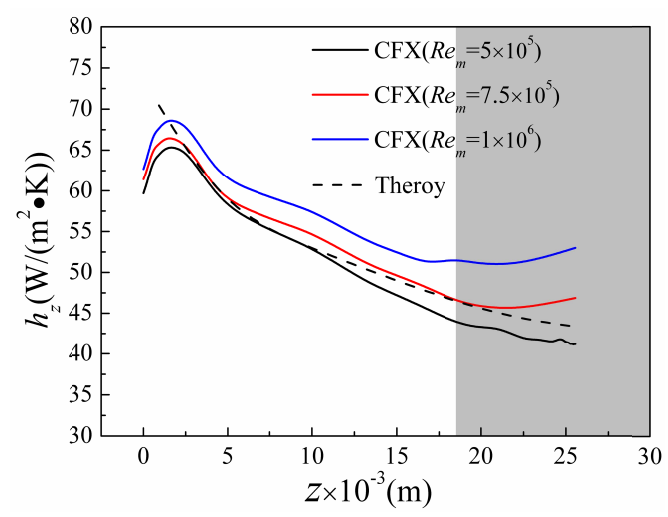

(b) Heat transfer on outer surface

Fig. 6 Effect of $R e_{m}$ on the heat transfer distributions $\left(R e_{\Phi}=4.65 \times 10^{5}, C_{w}=3008\right)$

Fig. 8 shows the turbine disk surface temperature profiles with different $R e_{m}$ and the corresponding temperature profiles are delineated in Fig. 7. It can be seen that the average temperature of turbine disk increases with the $R e_{m}$ increasing from $5 \times 10^{5}$ to $1 \times 10^{6}$. With the scope of this model turbine disk, there are four factors which can make effect on the temperature distribution of disk, they are: 1) the fluid reference temperature at the outer surface of turbine disk, 2) the heat transfer coefficient at the outer surface of turbine disk; 3) the fluid reference temperature at the side surface of turbine disk, 4) the heat transfer coefficient at the out surface of turbine disk. In this model of turbine disk, with the $R e_{m}$ increasing, the fluid reference temperature at the outer surface of turbine disk increases (as shown in Eq. 8). And 
there is a modest increase of the heat transfer coefficient in region A. As a consequence, the average temperature of the turbine disk increases. According to Fig.8a, the temperature deviation coefficient $\left(\varphi_{T}=\left(T_{t}-T_{s}\right) /\left(T_{m}-T_{c}\right)\right)$ is $3.9 \%$ for the case of $R e_{m}=5 \times 10^{5}$, while the $\varphi_{T}$ is increased to $5.5 \%$ and $7.2 \%$ at $R e_{m}=7.5 \times 10^{5}$ and $1 \times 10^{6}$ respectively. The explanations for this deviations may be found from Fig.6 (b), which shows a good agreement at $R e_{m}=7.5 \times 10^{5}$, and the obvious discrepancy for the case of $R e_{m}=1 \times 10^{6}$ and $R e_{m}=5 \times 10^{5}$ between the theoretical results and experimental data. Focusing on Fig. $8 \mathrm{~b}$, the theoretical temperature distributions are depicted and compared with the simulation results. Clearly, the theoretical temperature at disk outer surface increases with the increase of $R e_{m}$. Besides, there is a minor change in the axial direction ( $z$ direction), the maximum change range is less than $0.81 \%$ for $R e_{m}=7.5 \times 10^{5}$. Consequently, it is feasible to assume the disk outer surface temperature as the constant value in the axial direction.

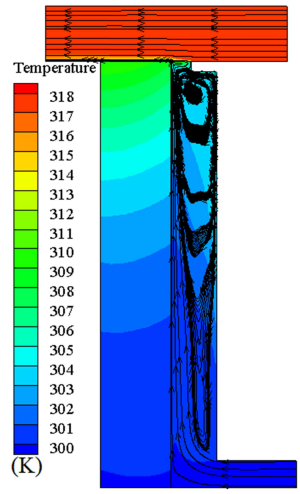

(a) $R e_{m}=5 \times 10^{5}$

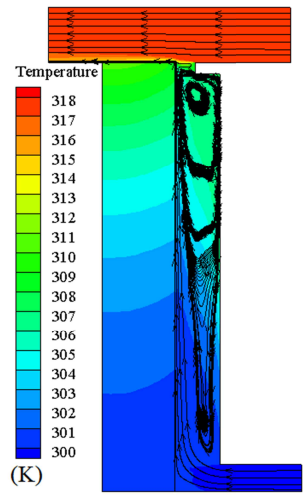

(b) $R e_{m}=7.5 \times 10^{5}$

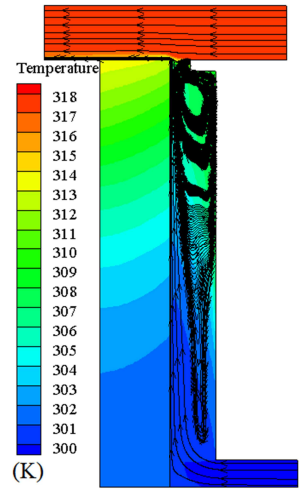

(c) $R e_{m}=1 \times 10^{6}$

Fig. 7 Distributions of disk temperature and streamline $\left(R e_{\Phi}=4.65 \times 10^{5}, C_{w}=3008\right)$

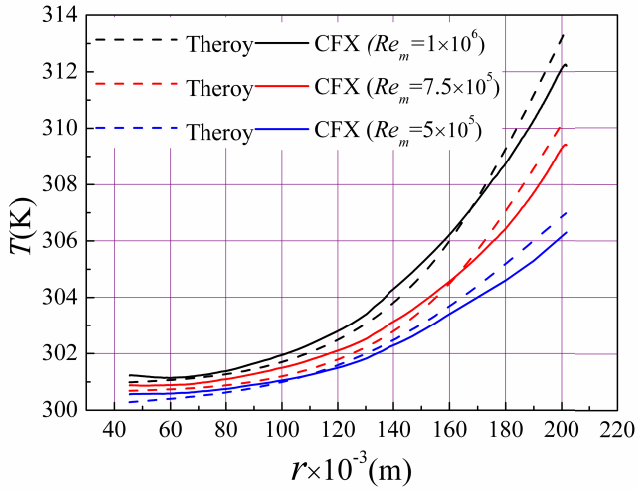

(a) Temperature distributions on side surface

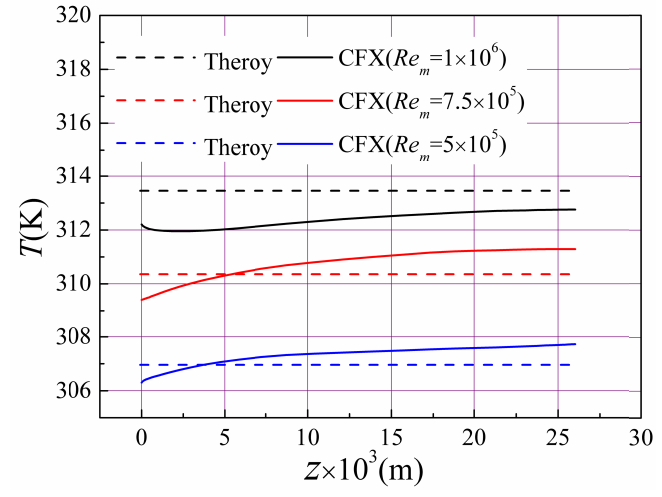

(b) Temperature distributions on outer surface

Fig. 8 Effect of $R e_{m}$ on the heat transfer distributions $\left(R e_{\Phi}=4.65 \times 10^{5}, C_{w}=3008\right)$

Fig.9 plots the effect of $C_{w}$ on the temperature profiles on the side surface and the outer surface of turbine disk, Inspection of Fig. 9a shows that the turbine disk temperature at side surface decreases with the increase of the non-dimensional mass flow of the cooling air, $C_{w}$. Note that the decrease of hot gas mass flow can also decrease the temperature of turbine disk as shown in Fig.8a. However, an obvious difference between two methods is the temperature change at low radius $(0.0455 \mathrm{~m}<r<0.12 \mathrm{~m})$, where the temperature change is minor for decreasing the $R e_{m}$ as shown in Fig.8a. On the other hand, there is a clear decrease of temperature while increasing the $C_{w}$ at low radius, hence, it can be concluded that improving the cooling air mass flow rate is more effective than reducing mainstream flow rate in reducing the disk center temperature. According to Fig 9b, the temperature distributions at turbine disk outer surface for $C_{w}=1504, C_{w}=3008$ and $C_{w}=7520$ are shown. Obviously, the temperature decreases with the increase of $C_{w}$, which is the same as Fig.9's. Besides, the theoretical results are in good agreement with simulation data for $C_{w}=1504$, with the maximum $\varphi_{T}, 2.2 \%$, which will increase to $8.7 \%$, while the $C_{w}$ increases to 7520 . The reason for this discrepancy is that the Eq. (2) does not consider the impact of cooling air mass flow, same as that of Fig.8b. Accordingly, this is also a future research direction, that is, how to utilize a correlation, which contains a clear physical meaning, to summarize the heat transfer characteristics at high radius (core region) and low radius (source region) at 
the same time.

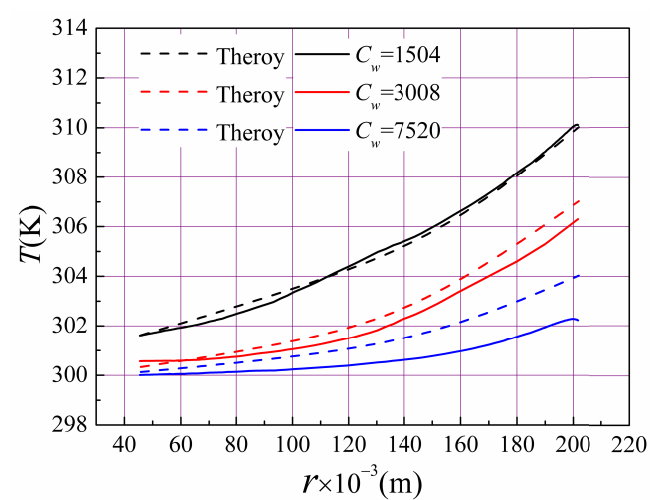

(a) Temperature distributions on side surface

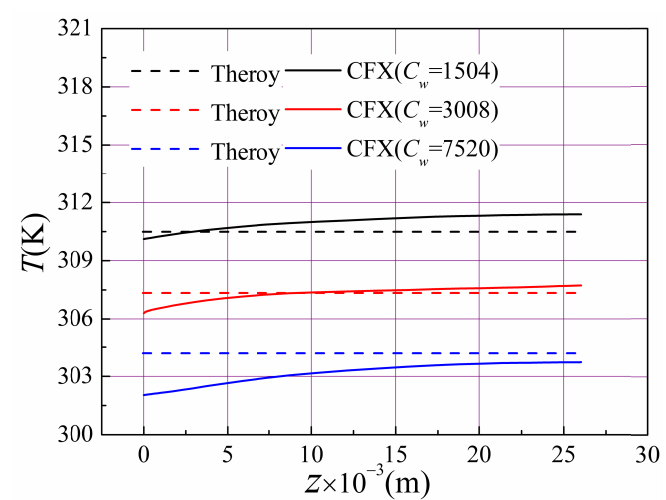

(b) Temperature distributions on outer surface

Fig. 9 Effect of $C_{w}$ on the heat transfer distributions $\left(R e_{\Phi}=4.65 \times 10^{5}, R e_{m}=5 \times 10^{5}\right)$

The temperature profiles at disk side surface are shown in Fig. 10a. It can be seen that the temperature at disk side surface has a modest increase with the increase of $R e_{\Phi}$. It can be concluded to two reasons which can make effect on the temperature distribution at disk side surface:1) the convective heat transfer coefficient at disk outer surface $\left(h_{r}\right)$ increases with the $R e_{\Phi}$ increasing, which means more heat is transferred to the disk, 2) the convective heat transfer coefficient at disk side surface $\left(h_{z}\right)$ increases with the $R e_{\Phi}$ increasing, which represents a better cooling of the disk. Based on the above two reasons, the temperature of turbine disk increases with the increase of rotating Reynolds number. Fig. $10 \mathrm{~b}$ illustrates the temperature profiles at disk outer surface for different $R e_{\Phi}$. The theoretical results are in good agreement with simulation data with $R e_{\Phi}=4.65 \times 10^{5}$, where the $\varphi_{T}$ is no more than $3.9 \%$. However, the $\varphi_{T}$ increases to $7.3 \%$ with the $R e_{\Phi}$ increasing to $8.6 \times 10^{5}$. It should be noted again that the discrepancy of $h_{r}$ between the theoretical results and simulation data increases with the $R e_{\Phi}$ increasing as shown in Fig. $4 \mathrm{a}$. Thus, it can be concluded that the temperature model in this paper is suited for computing the temperature distribution of turbine disk based on a good understanding of the heat transfer characteristics of disk, which can lay a foundation the thermal stress analysis of turbine disk.

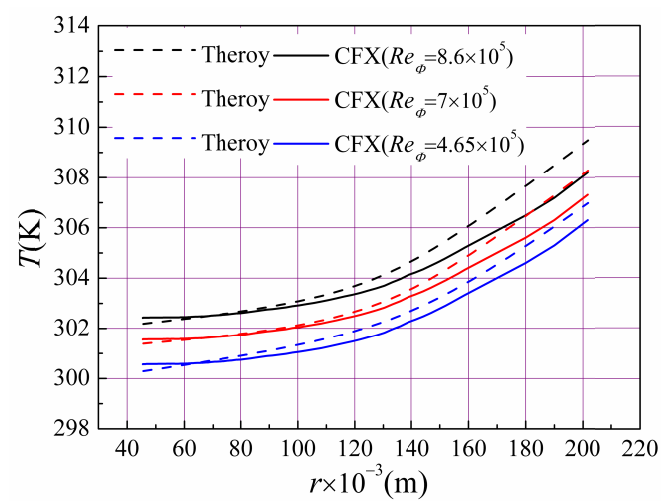

(a) Temperature distributions on side surface

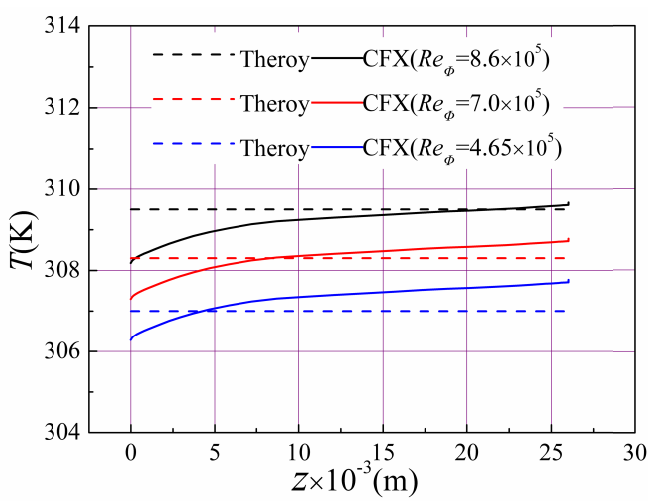

(b) Temperature distributions on outer surface

Fig. 10 Effect of $R e_{\Phi}$ on the heat transfer distributions $\left(C_{w}=3008, R e_{m}=5 \times 10^{5}\right)$

\section{Conclusions}

This paper presents a temperature coupling theoretical model of turbine disk, with the turbine disk temperature assumed to be axially constant, and the fluid flow at disk outer surface analogous to "fillm cooling". By using the Newton Rafael iterative method, the complete theoretical temperature distributions of turbine disk were obtained, and compared with the simulation results and part of experimental data. Some general inspections of this paper can be concluded as follow:

(1)The maximum $\varphi_{T}$ between the theoretical results (produced by this temperature coupling model) and simulation 
data is $8.7 \%$ for $R e_{\Phi}=4.65 \times 10^{5}, C_{w}=7520, R e_{m}=5 \times 10^{5}$. Hence, it is suitable for computing the temperature distribution of turbine disk by the analogy method of "film cooling".

(2)The convective heat transfer coefficient distributions at the disk outer surface exhibited the influence of the region $\mathrm{A}$ and the region $\mathrm{B}$. In the region $\mathrm{A}$, which is near to the outlet of cooling air, the convective heat transfer is dominated by the cooling air mass flow rate. In the core region, which is far from the outlet of cooling air, the heat transfer is dominated by the mainstream mass flow rate.

(3)The temperature of the turbine disk increases with the increase of the mainstream mass flow rate, and its affected region is mainly located in the high radius of the turbine disk. On the other hand, the overall temperature of turbine disk decreases with the increase of cooling air mass flow rate.

(4)With the increase of $R e_{\Phi}$, on the one hand, the convective heat transfer coefficient at disk outer surface $\left(h_{r}\right)$ increases with the $R e_{\Phi}$ increasing, which means more heat is transferred to the disk. On the other hand, the convective heat transfer coefficient at disk side surface $\left(h_{z}\right)$ increases with the $R e_{\Phi}$ increasing, which represents a better cooling effect of the disk. Finally, there is a minor temperature increase of turbine disk because of the comprehensive effect of the above two reasons.

\section{Acknowledgments}

The authors gratefully acknowledge the financial supports for this project from the Funding of Jiangsu Innovation Program for Graduate Education (grant No: KYLX16_0356), the Fundamental Research Funds for the Central Universities and the Funds for Research Institution in Shenyang.

\section{References}

Astarita, T. and Cardone. G., Convective heat transfer on a rotating disk with a centred impinging round jet, International Journal of Heat \& Mass Transfer, Vol.51, No.7-8 (2008), pp.1562-1572.

Batchelor, G.K., Note on a class of solutions of the navier-stokes equations representing steady rotationnally-symmetric flow, Quarterly Journal of Mechanics \& Applied Mathematics, Vol.4, No.1 (1951), pp.29-41.

Bunker, R.S., Metzger, D.E. and Wittig, S., Local heat transfer in turbine disk-cavities. I - Rotor and stator cooling with hub injection of coolant, Journal of Turbomachinery, Vol.114, No.1 (1992), pp.221-228.

Chen, Y. M., Lee, W.T. and Wu, S.J., Heat (mass) transfer between an impinging jet and a rotating disk, Heat \& Mass Transfer, Vol.34, No.2-3 (1998), pp.195-201.

Chew, J.W., Prediction of flow in rotating-disk systems using the k-epsilon turbulence model, ASME-AMER Soc. Mech. Eng. Mech. Eng, Vol.106 (1984), pp.82-93.

Daily, J.W. and Nece, R.E., Chamber dimension effects on induced flow and frictional resistance of enclosed rotating disks, Journal of Basic Engineering, Vol.82, No.1 (1960), pp.217-230.

Ding, S.T., Li, G. and Luo, B., Active control thermal-loading method to ameliorate stress in aeroengine turbine disk, Journal of Thermophysics \& Heat Transfer, Vol.27, No.2 (2013), pp.274-285.

Fenot, M., Vullierme, J. J. and Dorignac., E., Local heat transfer due to several configurations of circular air jets impinging on a flat plate with and without semi-confinement, International Journal of Thermal Sciences, Vol.44, No.7 (2005), pp.665-675.

Haynes, C.M. and Owen, J.M., Heat transfer from a shrouded disk system with a radial outflow of coolant, Journal of Engineering for Gas Turbines \& Power, Vol.97, No.1 (1975), pp.28-35.

Karman, T.V., Uber laminare und turbulente reibung, Journal of Applied Mathematics and Mechanics / Zeitschrift fur Angewandte Mathematik und Mechanik, Vol.1, No.4 (1921), pp.233-252.

Kilic, M., Computation of flow and heat transfer in rotating cavities with peripheral flow of cooling air, Annals of the New York Academy of Sciences, Vol.934, No.1 (2001), pp.513-520.

Lin, L., Wu, K., Tan, Q.X. and Ren, J., Heat transfer characteristics of rotor-stator cavity with small mass flow rate, Journal of Aerospace Power, Vol.30, No.9 (2015), pp.2058-2065 (in Chinese).

Luo, X., Zhao, X., Wang, L., Wu, H. and Xu, G., Flow structure and heat transfer characteristics in rotor-stator cavity with inlet at low radius, Applied Thermal Engineering, Vol.70, No.1 (2014), pp.291-306. 
Metzger, D.E., Carper, H.J. and Swank, L.R., Heat transfer with film cooling near nontangential injection slots, Journal of Engineering for Gas Turbines \& Power, Vol.90, No.2 (1968), pp.157-162.

Owen, J.M. and Bayley, F.J., Heat transfer from an air-cooled rotating disk, Proceedings of the Royal Society A, Vol.336, No.1607 (1974), pp.453-473.

Pelle, J. and Harmand, S., Heat transfer study in a rotor-stator system air-gap with an axial inflow, Applied Thermal Engineering, Vol.29, No.8-9 (2009), pp.1532-1543.

Pelle, J. and Harmand, S., Heat transfer measurements in an opened rotor-stator system air-gap, Experimental Thermal \& Fluid Science, Vol.31, No.3 (2007), pp.165-180.

Poncet, S. and Schiestel, R., Numerical modeling of heat transfer and fluid flow in rotor-stator cavities with throughflow, International Journal of Heat \& Mass Transfer, Vol.50, No.7 (2007), pp.1528-1644.

Roy, R.P., Xu, G. and Feng, J., A Study of Convective Heat Transfer in a Model Rotor-Stator Disk Cavity, Journal of Turbomachinery, Vol.123, No.3 (2001), pp.621-632.

Stewartson, K., On the flow between two rotating coaxial disks, Mathematical Proceedings of the Cambridge Philosophical Society, Vol.49, No.2 (1953), pp.333-341.

Wang, L. and Wilson, M.A., Effects of ingestion on the flow and heat transfer in a rotor-stator system, 17th Biennial Mogollon Archaeology Conference (2012), pp.129-130.

Yuan, Z.X., Saniei, N. and Yan, X.T., Turbulent heat transfer on the stationary disk in a rotor-stator system, International Journal of Heat \& Mass Transfer, Vol.46, No.12 (2003), pp.2207-2218. 